\title{
La percepción del buen profesor en universitarios: el caso de una universidad politécnica de México
}

\author{
The perception of the great teacher in university: the case of \\ A polytech university of mexico
}

A percepção de um bom professor em estudantes universitários: o caso de uma universidade politécnica no México

Daniela Cruz-Delgado

Universidad Politécnica de Victoria, México

dcruzd@upv.edu.mx https://orcid.org/0000-0003-0289-7483

Estela Torres-Ramírez

Universidad Politécnica de Victoria, México

etorresr@upv.edu.mx https://orcid.org/0000-0002-9601-7274

Juan López-Hernández

Universidad Politécnica de Victoria, México

jlopezh@upv.edu.mx https://orcid.org/0000-0002-9252-6338

Juan Enrique Lira-Uribe

Universidad Politécnica de Victoria, México

jlirau@upv.edu.mx https://orcid.org/0000-0002-3499-597X 


\section{Resumen}

Introducción: Los profesores universitarios son un factor clave para el desarrollo de los profesionales que requiere la sociedad. Por eso, es trascendental que las universidades cuenten con docentes competentes. Pero ¿cuáles son las características que debe reunir un buen profesor? Objetivo: Identificar las características y cualidades que definen al buen profesor desde la percepción de estudiantes de una universidad politécnica de México. Método: Se aplicó un cuestionario de 91 preguntas, con una sección de identificación general del encuestado y ocho dimensiones de las características y cualidades del buen profesor. El universo fueron 646 alumnos y la muestra probabilística estratificada por carreras impartidas de 241 elementos. Resultado: De las ocho dimensiones evaluadas por los estudiantes, las más importantes para definir a un buen profesor fueron las cualidades profesionales y las características de las explicaciones. Entre las cualidades profesionales, la competencia sobre su materia la perciben como la más importante; en otras palabras, el perfil del buen docente se basa en las competencias profesionales que posee, en qué tan bueno es para impartir su materia, qué tanto conoce los temas que aborda, y en su capacidad para trasmitir el conocimiento de modo que el alumno adquiera un aprendizaje significativo. Así, la competencia, la responsabilidad y la buena comunicación son los tres elementos de las características profesionales que los alumnos valoran del docente. Respecto a la dimensión características de la explicación (segunda más importante), la mayor puntuación fue para claridad de las explicaciones, así como uso de ejemplos prácticos y reales. Conclusiones: Si el docente cuenta con algunas de las características de un buen profesor, deberá seguir trabajando y fomentando sus fortalezas, así como sus debilidades a través de cursos y talleres de capacitación para optimizar sus actividades de enseñanza-aprendizaje.

Palabras clave: cualidades, desempeño, evaluación docente.

\section{Abstract}

Introduction: University professors are a key factor for the development of professionals required by society. For this reason, it is essential that universities have competent professors. But are they the functions that a good teacher should meet? Objective: Identify the characteristics and qualities that define a good teacher from the perception of students from a polytechnic university in Mexico. Method: A 91-question questionnaire was applied, with a general identification section of the respondent and eight dimensions of the characteristics and qualities of a good teacher. The universe consisted of 646 students and the probabilistic sample stratified by courses taught with 241 elements. Result: Of the eight dimensions evaluated by 
the students, the professional qualities and the characteristics of the explanations are perceived as the most important to define a good teacher. Among the professional qualities, the competence on their subject is perceived as the most important, in this way, the profile of a good teacher is based on the professional skills they have, how good they are at teaching their subject, how much they know and know of the topics it addresses and in which it has the ability to transmit knowledge optimally so that the student acquires meaningful learning. Thus, competence, responsibility and good communication are the three elements of the professional characteristics that students value from the teacher. Regarding the dimension of explanation characteristics, the second most important, the clarity of the explanations, as well as the use of practical and real examples, resulted with the highest score. Conclusions: If the teacher has some of the characteristics that make the teacher a good teacher, they should continue to work and promote the strengths that they identify in their person and improve their weaknesses through courses and training workshops, in such a way that it increases its potential and quality, which must be manifested in the teaching-learning process.

Keywords: qualities, performance, teacher evaluation.

\section{Resumo}

Introdução: O professor universitário é um fator fundamental para o desenvolvimento dos profissionais que a sociedade exige. Portanto, é essencial que as universidades tenham professores competentes. Mas quais são as características que um bom professor deve ter? Objetivo: Identificar as características e qualidades que definem um bom professor a partir da percepção dos alunos de uma universidade politécnica no México. Método: Foi aplicado um questionário de 91 questões, com uma seção de identificação geral do respondente e oito dimensões das características e qualidades de um bom professor. O universo era de 646 alunos e a amostra probabilística estratificada por carreiras ensinou 241 elementos. Resultado: Das oito dimensões avaliadas pelos alunos, as mais importantes para definir um bom professor foram as qualidades profissionais e as características das explicações. Dentre as qualidades profissionais, a competência na matéria é percebida como a mais importante; Em outras palavras, o perfil de um bom professor é baseado nas competências profissionais que possui, quão bom ele é no ensino da matéria, quão bem conhece os temas que aborda e sua capacidade de transmitir conhecimentos para que o aluno adquira uma aprendizagem significativa. Assim, competência, responsabilidade e boa comunicação são os três elementos das características profissionais que os alunos valorizam do professor. Em relação à dimensão características da explicação (a segunda mais importante), a maior pontuação foi para a clareza das explicações, 
bem como a utilização de exemplos práticos e reais. Conclusões: Se o professor possui algumas das características de um bom professor, ele deve continuar trabalhando e promovendo seus pontos fortes, assim como seus pontos fracos, por meio de cursos de capacitação e oficinas para otimizar suas atividades de ensino-aprendizagem.

Palavras-chave: qualidades, desempenho, avaliação de professores.

Fecha Recepción: Agosto 2020

Fecha Aceptación: Mayo 2021

\section{Introducción}

Los profesores universitarios son un factor clave para el desarrollo de los profesionales que requiere la sociedad, de ahí que sea trascendental que las universidades cuenten con docentes competentes. Pero ¿cuáles son las cualidades que debe reunir un buen profesor? La respuesta a esta interrogante variará dependiendo del grupo al que se le plantee, aunque será a los propios docentes a quienes más les interesará conocer los aspectos que se deben tomar en cuenta para optimizar la práctica pedagógica.

Desde el punto de vista normativo, son diversos los organismos encargados de fomentar la educación de la población, derecho humano que transforma vidas, como lo señala la Organización de las Naciones Unidas para la Educación, la Ciencia y la Cultura (Unesco, 2020). Por eso, en mayo de 2015, dentro del Foro Mundial sobre la Educación, la comunidad educativa global se comprometió a "asegurar que los profesores sean adecuadamente contratados, empoderados, bien formados, profesionalmente capacitados, motivados y apoyados por sistemas con recursos, eficientes y bien regulados" (Unesco, 2015, p. 3). Con ello, se busca mejorar la calidad de la educación, elemento esencial para contribuir a la solución de problemas como la pobreza y el hambre.

En tal sentido, en la Asamblea General de la Organización de las Naciones Unidas (ONU) realizada en septiembre de 2015, los dirigentes mundiales aprobaron la Agenda 2030, la cual consta de 17 objetivos del desarrollo sostenible (ODS) que buscan transformar el mundo. El objetivo cuatro, por ejemplo, se enfoca en "garantizar una educación inclusiva, equitativa y de calidad", mientras que la meta 4.C procura que para el año 2030 aumente "sustancialmente la oferta de maestros calificados, incluso mediante la cooperación internacional para la formación de docentes en países en desarrollo, especialmente los países menos adelantados y los pequeños Estados insulares en desarrollo" (ONU, 2015, pp. 19-20).

Con el propósito de apoyar la consecución de esta meta sobre profesores, el Equipo Especial Internacional sobre Docentes para la Educación para Todos (EPT), en estrecha coordinación con las entidades de la Unesco y los socios externos del Equipo Especial, elaboraron la Guía para el Desarrollo de Políticas Docentes, donde se presentan lo que 
consideran como las dimensiones más importantes para la política docente:

1. Contratación y retención de docentes.

2. Educación de los docentes (inicial y continua).

3. Distribución.

4. Estructuras/trayectorias profesionales.

5. Empleo y condiciones laborales de los docentes.

6. Recompensas y remuneraciones para docentes.

7. Estándares docentes.

8. Responsabilidad de los docentes.

9. Gestión escolar.

Estas nueve dimensiones son claves para cualquier política docente integral, aunque también vale subrayar la necesidad de conocer la percepción del alumno sobre la práctica pedagógica para identificar aspectos mejorables de la educación.

La presente investigación, por tanto, se enfoca en los puntos siete y ocho listados anteriormente, aunque examinados desde la percepción de alumnos de nivel superior que cursan el último ciclo de formación en una universidad politécnica de México, nivel educativo que articula el crecimiento tecnológico, científico y económico de un país.

Los estándares docentes y la responsabilidad de ellos son elementos que deben considerarse en los procesos de selección y contratación de profesores universitarios, de ahí que exista la necesidad de precisar las características y cualidades que debe tener el buen docente. En este sentido, el Plan Nacional de Desarrollo 2013-2018, en México, hace suya la prioridad de la educación de calidad al incluirla como una de sus cinco metas nacionales, donde señala que las posibilidades de desarrollo del país dependen de una educación de calidad. Este propósito es coherente con lo planteado en la Ley General de Educación, cuya meta es garantizar el derecho de acceso a la educación establecido en la Constitución Política de los Estados Unidos Mexicanos, reconocido en su artículo 3 por el bienestar que confiere a todas las personas (Diario Oficial de la Federación [DOF], 2021). En pocas palabras, la educación superior del país busca propiciar a través de políticas y programas de apoyo las condiciones necesarias para que la sociedad mexicana reciba una formación universitaria de calidad (Secretaría de Educación Pública [SEP], 2011).

En este contexto, existen diversas investigaciones que analizan los factores que hacen que los docentes impartan educación de calidad. Por ejemplo, Miranda (2007) señala que la calidad de vida de un país se encuentra muy ligada a la calidad de su sistema educativo, espacio donde el docente tiene un rol esencial, ya que es quien todos los días forma a las nuevas generaciones. Los profesores, por tanto, son un factor determinante en la calidad de los 
resultados del proceso de enseñanza-aprendizaje, cuyo objetivo primordial es el desarrollo de competencias del alumnado a través de enfoques educativos flexibles que busquen responder a los requerimientos del proceso productivo, a las formas de organización laboral, a las nuevas tecnologías de la información y a la actualización permanente e innovadora de toda profesión (Torres-Rivera, Badillo-Gaona, Valentin-Kajatt y Ramírez-Martínez, 2014).

Asimismo, Ruiz-Bueno, Mas-Torelló, Tejeda-Fernández y Navío-Gámez (2008) señalan que el profesor de educación superior pasa de ser un transmisor de conocimientos a un facilitador, tutor y gestor de aprendizajes, mientras que Cardona-Rodríguez, BarrenetxeaAyesta, Mijangos-del-Campo y Olaskoaga-Larrauri (2009) consideran que todo profesor debe conocer y desarrollar nuevos roles distintos de los tradicionales en el aula, como el liderazgo directivo, transaccional, transformacional, compartido y también la negociación de los conflictos.

Por su parte, Santos-Guerra (1991) menciona que el profesor de nivel superior no solo debe dominar su disciplina, sino también saber qué sucede en el aula y cómo aprenden los alumnos para implementar estrategias de intervención oportunas según cada contexto. En tal sentido, Hamer-Flores (2013), Tarabay-Yunes (2009) y Rocha-Chávez (2012) lograron identificar una serie de características que debe reunir un profesor competente: abierto a la comunicación con el alumnado, organizado en sus clases, claro en sus explicaciones, abierto a aceptar correcciones de los alumnos, capaz de valorar el esfuerzo del alumno y de usar un sistema de evaluación continua, puntual y exigente, entre otras.

Ahora bien, desde la visión de los estudiantes, se puede decir que estos califican a un buen profesor desde diferentes ópticas. Cabalín-Silva, Navarro-Hernández y Zamora-Silva (2010) señalan que para los alumnos que cursan el último año curricular los atributos esenciales del profesor universitario deben ser responsabilidad, respetuosidad y empatía, inteligencia, puntualidad, amabilidad, comprometido y motivador.

Sayós, Pagés, Amador y Jorba (2014), Abadía-Valle et al. (2015) y Merellano-Navarro, Almonacid-Fierro, Moreno-Doña y Casto-Jaque (2016) coinciden en que un profesor universitario es el gestor del conocimiento y las actividades de aprendizaje, de ahí que deba ser creativo, con capacidad para innovar, socializador y generador de actitudes; asimismo, un modelo profesional y humano a imitar, que concibe el aprendizaje como algo emocional y no solo cognitivo, capaz de trabajar en grupo y utilizar adecuadamente las nuevas tecnologías, así como de gestionar e investigar sobre su propia docencia. Es decir, el alumnado no solo valora la competitividad y profesionalismo del profesor como pedagogo, sino también muestra interés por los rasgos interpersonales, formativos-pedagógicos, humanísticos-emocionales y éticomorales, destacando en todo momento la centralidad en el aprendizaje de los estudiantes 


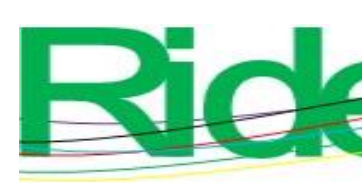

Revista Iberoamericana para la Investigación y el Desarrollo Educativo ISSN 2007 - 7467

(Gutiérrez, 2017).

Por su parte, Hickman, Alarcón, Cepeda, Cabrera y Torres (2016) indican que los estudiantes valoran principalmente el conocimiento del docente, lo que sustenta la idea de que el buen profesorado incorpora en su práctica docente los conocimientos profesionales (Vaillant y Siqueira, 2017), mientras que Yurén, García-García, Escalante Ferrer, González-Barrera y Velázquez Albavera (2020) señalan que los profesores estiman más las formas de relacionarse con los alumnos, y Valencia (2019) señala que "lo que anhela la sociedad son hombres y mujeres entregados a sus pasiones y que sepan transmitirlas" (p. 10).

Ahora bien, conscientes de que los profesores son los principales actores frente a los alumnos, consideramos que resulta de suma importancia evaluar al docente basado en la percepción de los estudiantes. Por ello, el objetivo general de este estudio fue identificar los atributos y cualidades que caracterizan a un buen profesor desde la percepción de los alumnos del último ciclo de formación de una universidad politécnica de México. De este modo se procura encontrar áreas de oportunidad para elevar su potencial y calidad en el proceso de enseñanza-aprendizaje.

La hipótesis planteada fue que las características profesionales son las que los estudiantes consideran de mayor importancia, ya que de ellas depende la competencia del profesor para impartir efectivamente su cátedra y para generar el aprendizaje significativo. Para comprobar la hipótesis se aplicó el método de la encuesta, a través de un cuestionario estructurado, con escala tipo Likert, como se detalla a continuación.

\section{Metodología}

\section{Descripción de la unidad de análisis}

La Universidad Politécnica de Victoria (UPV) cuenta con una oferta educativa centrada en cinco especialidades: Ingeniería Mecatrónica, Ingeniería en Tecnologías de la Información, Ingeniería en Tecnologías de Manufactura, Ingeniería en Sistemas Automotrices y la Licenciatura en Administración y Gestión de Pequeñas y Medianas Empresas. Ofrece además los programas de maestría en Ingeniería y maestría en Energías Renovables, lo que permite satisfacer la demanda de los sectores industrial, comercial y de servicios.

El papel del profesor en la universidad es decisivo para el cumplimiento de los planes y programas de estudio; ello le obliga a reflexionar sobre sus propias competencias profesionales y sus habilidades docentes para llevar cabo el proceso de enseñanza-aprendizaje. El modelo educativo de la universidad concibe al profesor como un agente de renovación y cambio, como facilitador y mediador entre el conocimiento y el aprendizaje del alumno. A través de su labor 


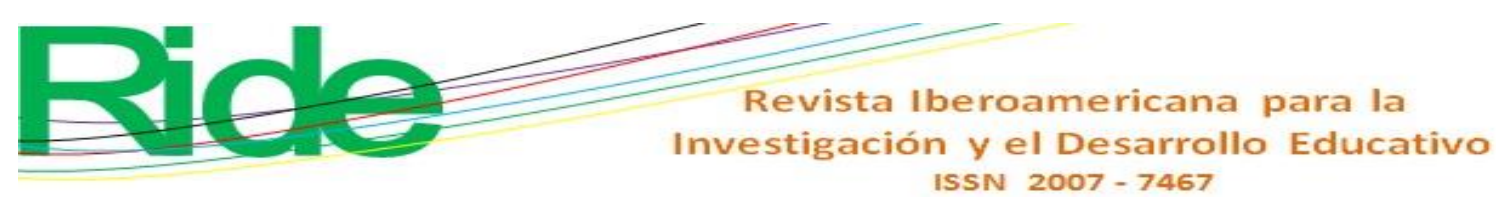

docente, los profesores contribuyen a que los alumnos aprendan a aprender.

El perfil del profesor considera cuatro dimensiones necesarias para cumplir con el desarrollo de las competencias establecidas en los perfiles profesionales: dominio de su disciplina (experiencia laboral, investigación y desarrollo tecnológico, y preservación y difusión de la cultura), características personales (capacidades como saber oír y aceptar sugerencias, saber determinar con claridad las capacidades asociadas a cada unidad de aprendizaje, establecer compromisos y cumplirlos, y propiciar ambientes que faciliten el aprendizaje y la apropiación de competencias), competencias docentes (competencias para el manejo de técnicas de aprendizaje que motiven a los alumnos para que, a partir de su experiencia, impriman un significado distinto a la enseñanza, es decir, que tengan vocación para enseñar y para aprender de los alumnos, en un proceso de enriquecimiento mutuo) y competencias tecnológicas (relacionadas con el uso de software educativo, presentaciones electrónicas y disposición para aplicar las diversas tecnologías educativas).

\section{Instrumento para recabar la información}

A través del método de la encuesta, se aplicó un cuestionario como instrumento de obtención de información a estudiantes de las cinco carreras que se ofertan y que se encontraban cursando el último ciclo de formación de una universidad politécnica. Las universidades politécnicas en México cuentan con un plan de estudios dividido de diez cuatrimestres. La población en estudio corresponde al último ciclo de formación, es decir, alumnos de séptimo a décimo cuatrimestre. Se omitieron en el universo de estudio a los estudiantes de los primeros cuatrimestres porque gran cantidad de ellos provienen del modelo educativo tradicional, y no del modelo basado en competencias; por ello, el objetivo tuvo implícito identificar la percepción de los estudiantes inmersos en este modelo educativo.

Se dispuso de 646 alumnos, y se seleccionó una población de prueba de 241 estudiantes a través de muestreo probabilístico estratificado; los estratos fueron definidos por las carreras a las que pertenecen los estudiantes (tabla 1). Se asume un margen de error de $5 \%$ y un nivel de confianza de $95 \%$. El análisis de fiabilidad del instrumento dio como resultado un valor de alfa de Cronbach de 0.923 . 
Tabla 1. Muestra estratificada por carrera

\begin{tabular}{|l|c|c|c|c|}
\hline Especialidad & Frecuencia & Porcentaje & $\begin{array}{c}\text { Porcentaje } \\
\text { válido }\end{array}$ & $\begin{array}{c}\text { Porcentaje } \\
\text { acumulado }\end{array}$ \\
\hline Pymes & 65 & 27.0 & 27.0 & 27.0 \\
\hline ITI & 60 & 24.9 & 24.9 & 51.9 \\
\hline ITM & 37 & 15.4 & 15.4 & 67.2 \\
\hline IM & 58 & 24.1 & 24.1 & 91.3 \\
\hline ISA & 21 & 8.7 & 8.7 & 100.0 \\
\hline Total & 241 & 100.0 & 100.0 & \\
\hline
\end{tabular}

Fuente: Elaboración propia

El cuestionario estuvo integrado por una sección de identificación del encuestado (cinco reactivos) y ocho dimensiones (tabla 2) sobre las cualidades del buen profesor (91 reactivos), de acuerdo con Hamer-Flores (2015), quedando estructurado con 91 reactivos, integrados por dimensión con la cantidad de reactivos que se indican en la tabla 2. La escala utilizada en el instrumento fue tipo Likert de cinco puntos ( $1=$ muy poco importante y $5=$ muy importante). La encuesta se aplicó en las aulas de los estudiantes, previa autorización de los docentes.

Tabla 2. Dimensiones de las características y cualidades de un buen profesor

\begin{tabular}{|l|c|}
\hline \multicolumn{1}{|c|}{ Dimensión } & $\begin{array}{c}\text { Cantidad } \\
\text { de } \\
\text { reactivos }\end{array}$ \\
\hline Cualidades personales del profesor & 14 \\
\hline Cualidades profesionales del profesor & 9 \\
\hline Características de la metodología de enseñanza del buen profesor & 8 \\
\hline Características de las explicaciones del buen profesor & 14 \\
\hline Los métodos que debería utilizar el buen profesor & 15 \\
\hline Materiales y recursos que debería utilizar el buen profesor & 9 \\
\hline Métodos de evaluaciones que debería utilizar el buen profesor & 13 \\
\hline Características de la evaluación que debería utilizar el buen profesor & 9 \\
\hline Total & 91 \\
\hline
\end{tabular}

Fuente: Elaboración propia

La información se procesó y analizó con el software de IBM Statistical Package for Social Science (SPSS), versión 20. Las técnicas de análisis de datos consistieron en análisis de frecuencias, diagrama de barras y análisis de varianza de un factor (Anova). 


\section{Resultados}

El género de los estudiantes del último ciclo de formación fue $60.2 \%$ masculino y $38.2 \%$ femenino ( $1.6 \%$ de la población no contestó). Como se muestra en la tabla 3 , de los cinco programas educativos, en cuatro de ellos prevalecen estudiantes del género masculino, y solo en la licenciatura en Administración y Gestión de Pymes predomina el femenino debido, probablemente, a estereotipos culturales que establecen que existen profesiones exclusivas para mujeres y para hombres, aunque esto se deberá profundizar en una futura investigación.

Tabla 3. Género de los estudiantes por carrera

\begin{tabular}{|l|l|c|c|c|c|c|c|}
\hline \multirow{2}{*}{} & \multicolumn{5}{|c|}{ Carrera } & \multirow{2}{*}{ Total } \\
\cline { 2 - 8 } \multicolumn{2}{|c|}{} & Pymes & ITI & ITM & IM & ISA & 145 \\
\hline \multirow{2}{*}{ Género } & Masculino & 22 & 35 & 21 & 48 & 19 & 92 \\
\cline { 2 - 8 } & Femenino & 43 & 23 & 15 & 9 & 2 & 237 \\
\hline Total & 65 & 58 & 36 & 57 & 21 & 27 \\
\hline
\end{tabular}

Fuente: Elaboración propia

Los estudiantes encuestados consideran que las cualidades personales más importantes que posee un buen profesor son el respeto hacia los alumnos, la honestidad y la capacidad de escucha; por el contrario, la simpatía y el ser divertido son menos importantes (tabla 4). 
Tabla 4. Grado promedio de importancia que otorgan los estudiantes a las cualidades personales del buen profesor

\begin{tabular}{|l|c|}
\hline \multicolumn{1}{|c|}{ Cualidades personales del buen profesor } & Media \\
\hline 1. Simpatía & 3.8 \\
\hline 2. Buen humor & 4.0 \\
\hline 3. Carácter agradable & 4.2 \\
\hline 4. Divertido & 3.4 \\
\hline 5. Abierto a los alumnos & 4.6 \\
\hline 6. Comprensivo & 4.4 \\
\hline 7. Honesto & 4.7 \\
\hline 8. Buena persona & 4.3 \\
\hline 9. Que respete a los alumnos & 4.8 \\
\hline 10. Con buen trato & 4.6 \\
\hline 11. Con empatía & 4.3 \\
\hline 12. Con capacidad de escucha & 4.7 \\
\hline 13. Que dé confianza & 4.5 \\
\hline 14. Atento & 4.4 \\
\hline Media global & 4.3 \\
\hline
\end{tabular}

Fuente: Elaboración propia

Las cualidades profesionales fueron calificadas con las puntuaciones más altas por los estudiantes. La competencia sobre su materia la perciben como muy importante, seguida de la responsabilidad y la buena comunicación (tabla 5). 
Tabla 5. Grado promedio de importancia que otorgan los estudiantes a las cualidades profesionales del buen profesor

\begin{tabular}{|l|c|}
\hline \multicolumn{1}{|c|}{ Cualidades profesionales del buen profesor } & Media \\
\hline 1. Competencia. Sabe su materia & 4.9 \\
\hline 2. Responsabilidad & 4.8 \\
\hline 3. Seriedad & 4.0 \\
\hline 4. Inteligencia & 4.6 \\
\hline 5. Buena comunicación & 4.7 \\
\hline 6. Puntualidad & 4.3 \\
\hline 7. Experiencia profesional & 4.4 \\
\hline 8. Prepara las clases & 4.5 \\
\hline 9. Respeta los horarios de tutoría y asesoría & 4.3 \\
\hline Media global & 4.5 \\
\hline
\end{tabular}

Fuente: Elaboración propia

Respecto a la metodología de enseñanza, las características que fueron calificadas de mayor importancia para los estudiantes fueron establecer relaciones entre los conceptos y temas de la asignatura (tabla 6).

Tabla 6. Grado promedio de importancia que otorgan los estudiantes a la metodología de enseñanza del buen profesor

\begin{tabular}{|l|c|}
\hline \multicolumn{1}{|c|}{ Metodología de enseñanza } & Media \\
\hline 1. Parte de lo aprendido en cursos anteriores & 4.2 \\
\hline 2. Establece relaciones entre los conceptos y temas de la asignatura & 4.7 \\
\hline $\begin{array}{l}\text { 3. Enseña estrategias para trabajar la asignatura y aprender (técnicas de estudio, } \\
\text { preparación de exámenes, hablar en público...) }\end{array}$ & 4.4 \\
\hline 4. Fomenta la participación e implicación de los alumnos & 4.3 \\
\hline 5. Promueve el trabajo individual del alumno & 4.0 \\
\hline 6. Utiliza una metodología diversa y adapta a las características de los alumnos & 4.3 \\
\hline 7. Reduce la clase magistral a lo estrictamente necesario & 3.9 \\
\hline 8. Utiliza los recursos de apoyo necesarios (Power Point, videos...) & 4.3 \\
\hline Media global & 4.3 \\
\hline
\end{tabular}

Fuente: Elaboración propia 
La calidad de las explicaciones es una de las características que los estudiantes consideran de mayor importancia en un buen profesor, pues de ello depende que su aprendizaje sea significativo y que comprendan los temas abordados en clase (tabla 7).

Tabla 7. Grado promedio de importancia que otorgan los estudiantes a la calidad de la explicación del buen profesor

\begin{tabular}{|l|c|}
\hline \multicolumn{1}{|c|}{ Calidad de la explicación } & Media \\
\hline 1. Claras & 4.9 \\
\hline 2. Sencillas y con vocabulario comprensible & 4.6 \\
\hline 3. Precisas & 4.6 \\
\hline 4. Amenas & 4.3 \\
\hline 5. Divertidas & 3.8 \\
\hline 6. Motivadoras & 4.3 \\
\hline 7. Interesantes & 4.7 \\
\hline 8. Haciendo uso de ejemplos prácticos y reales & 4.7 \\
\hline 9. Destacando los conceptos básicos & 4.4 \\
\hline 10. Con buen orden & 4.4 \\
\hline 11. Adecuadas a un ritmo, dando tiempo para el aprendizaje & 4.5 \\
\hline 12. Con volumen y tono de voz adecuado & 4.5 \\
\hline 13. Dedica un tiempo breve a recordar aprendizajes de las clases anteriores & 4.3 \\
\hline 14. Relaciona teoría y práctica & 4.6 \\
\hline Media global & 4.5 \\
\hline
\end{tabular}

Fuente: Elaboración propia

Los diversos métodos didácticos utilizados por los docentes en el aula son determinantes en la adquisición del conocimiento por parte de los alumnos. La resolución de dudas es un factor de gran importancia y, en consecuencia, una de las características que mejor debe trabajar todo profesor (tabla 8). 
Tabla 8. Grado promedio de importancia que otorgan los estudiantes a los métodos didácticos empleados por el buen profesor

\begin{tabular}{|c|c|}
\hline Métodos didácticos & Media \\
\hline 1. Método socrático-mayéutico & 4.4 \\
\hline 2. Análisis de casos & 4.1 \\
\hline 3. Aprendizaje por descubrimiento & 3.9 \\
\hline $\begin{array}{l}\text { 4. Aprendizaje significativo (no memorístico, estableciendo relaciones entre lo } \\
\text { nuevo y lo viejo) }\end{array}$ & 4.3 \\
\hline 5. Uso de esquemas y resúmenes en la pizarra que son explicados luego & 3.9 \\
\hline 6. Uso de preguntas referidas a los contenidos para que el alumno participe & 4.1 \\
\hline 7. Lección magistral & 3.6 \\
\hline 8. Seminarios & 3.5 \\
\hline 9. Explicación de un contenido de diversas maneras & 4.2 \\
\hline 10. Resolución de dudas en clase & 4.9 \\
\hline 11. Resolución de dudas en tutoría y asesoría & 4.5 \\
\hline 12. Método tradicional & 4.3 \\
\hline 13. Trabajo sobre temas de actualidad & 4.5 \\
\hline 14. Exigencia de lecturas previas de los alumnos & 3.9 \\
\hline 15. Exigencia de exposiciones orales de los alumnos & 3.7 \\
\hline Media global & 4.1 \\
\hline
\end{tabular}

\section{Fuente: Elaboración propia}

Los materiales de estudio claros y sencillos resultan de mayor importancia para los estudiantes, seguidos de apuntes que se ajusten al aprendizaje que debe obtener el alumno (tabla 9). 
Tabla 9. Grado promedio de importancia que otorgan los estudiantes a los materiales y recursos didácticos utilizados por el buen profesor

\begin{tabular}{|l|c|}
\hline \multicolumn{1}{|c|}{ Materiales y recursos didácticos } & Media \\
\hline 1. Material de estudio claro y sencillo & 4.6 \\
\hline 2. Referencias bibliográficas precisas & 4.1 \\
\hline 3. Apuntes de calidad ajustados a lo que hay que aprender & 4.4 \\
\hline \begin{tabular}{l} 
4. Apuntes en la Web para no tener que copiar todo el tiempo \\
\hline 5. Modelos de exámenes
\end{tabular} & 4.0 \\
\hline $\begin{array}{l}\text { 6. Ejercicios resueltos } \\
\text { multimedia, pizarra, etc.) }\end{array}$ & 4.0 \\
\hline $\begin{array}{l}\text { 7. Medios audiovisuales (videos, gráficos, diapositivas tradicionales, } \\
\text { relacionadas, etc.) }\end{array}$ & 3.7 \\
\hline $\begin{array}{l}\text { 9. Uso de material complementario para el aprendizaje (esquemas, } \\
\text { resúmenes, fotocopias) }\end{array}$ & 3.9 \\
\hline \begin{tabular}{l} 
Media global \\
\hline
\end{tabular} & 4.1 \\
\hline
\end{tabular}

\section{Fuente: Elaboración propia}

Para los estudiantes encuestados es primordial la valoración que da el profesor a la realización de prácticas y al esfuerzo del alumno. Resultó de menor importancia dentro de los métodos de evaluación la aplicación de un solo examen final (tabla 10). La valoración de la asistencia tanto a clase como a tutorías y asesorías para la resolución de dudas son aspectos poco valorados. 
Tabla 10. Grado promedio de importancia que otorgan los estudiantes a los métodos de evaluación aplicados por el buen profesor

\begin{tabular}{|l|c|}
\hline \multicolumn{1}{|c|}{ Métodos de evaluación } & Media \\
\hline 1. Solo examen final & 2.5 \\
\hline 2. Exámenes parciales & 3.9 \\
\hline 3. Valoración de trabajos & 4.3 \\
\hline 4. Valoración de la asistencia a clase & 3.4 \\
\hline 5. Valoración de la asistencia a las tutorías y asesorías & 3.0 \\
\hline 6. Valoración de las actividades diarias de clase & 4.0 \\
\hline 7. Valoración del esfuerzo del alumno & 4.3 \\
\hline 8. Valoración del interés del alumno & 4.3 \\
\hline 9. Valoración de la realización de prácticas & 4.4 \\
\hline 10. Valoración de un portafolios & 3.4 \\
\hline 11. Valoración de resúmenes & 3.4 \\
\hline 12. Ausencia de exámenes y sustituciones por otros métodos & 3.7 \\
\hline 13. Realizar un proyecto y defenderlo al final del curso & 4.3 \\
\hline Media global & 3.8 \\
\hline
\end{tabular}

\section{Fuente: Elaboración propia}

Dar a conocer los criterios y procedimientos de evaluación es muy valorado por los estudiantes (tabla 11). Al respecto, Nowakowski (2007) y Abadía-Valle et al. (2015) destacan la importancia de cualidades como el profesionalismo, la escrupulosidad y la objetividad, así como el utilizar procedimientos de evaluación coherentes con los objetivos de la asignatura. En otras palabras, si los alumnos están informados sobre lo que se espera de ellos, pueden también definir sus estrategias individuales de estudio. 
Tabla 11. Grado promedio de importancia que otorgan los estudiantes a las características de la evaluación realizada por el buen profesor

\begin{tabular}{|l|c|}
\hline \multicolumn{1}{|c|}{ Características de la evaluación } & Media \\
\hline 1. Continua (no un único examen final) & 4.2 \\
\hline 2. Formativa & 4.5 \\
\hline 3. Justa, adecuada a lo trabajado en la asignatura & 4.5 \\
\hline 4. Flexible, que ofrezca opciones diversas para aprobar & 4.3 \\
\hline 5. Que exija lo básico, que no exija demasiado & 3.7 \\
\hline 6. Con elevada exigencia para el alumno & 3.6 \\
\hline 7. Negociando entre los alumnos y el profesor & 3.5 \\
\hline 8. Valorando no solo la memorización, sino el razonamiento y el aprendizaje & 4.5 \\
\hline significativo & 4.6 \\
\hline 9. Que los alumnos conozcan los criterios y procedimientos de evaluación & 4.2 \\
\hline Media global & \\
\hline
\end{tabular}

Fuente: Elaboración propia

Hasta aquí se analizan los resultados obtenidos para cada una de las dimensiones de las competencias y cualidades que caracterizan a un buen profesor. La figura 1 muestra el grado promedio de importancia que los estudiantes perciben que tiene cada una de las dimensiones y que debería tener el buen profesor. Las cualidades profesionales y las características de las explicaciones se perciben como las más importantes para definir a un buen profesor. El perfil del buen docente se basa en las competencias profesionales que posee, en qué tan bueno es para impartir su materia, qué tanto sabe y conoce de los temas que aborda y en que tenga la capacidad de trasmitir el conocimiento de forma óptima de tal manera que el alumno adquiera aprendizaje significativo. 
Figura 1. Grado promedio de importancia de las dimensiones sobre las características de un buen profesor

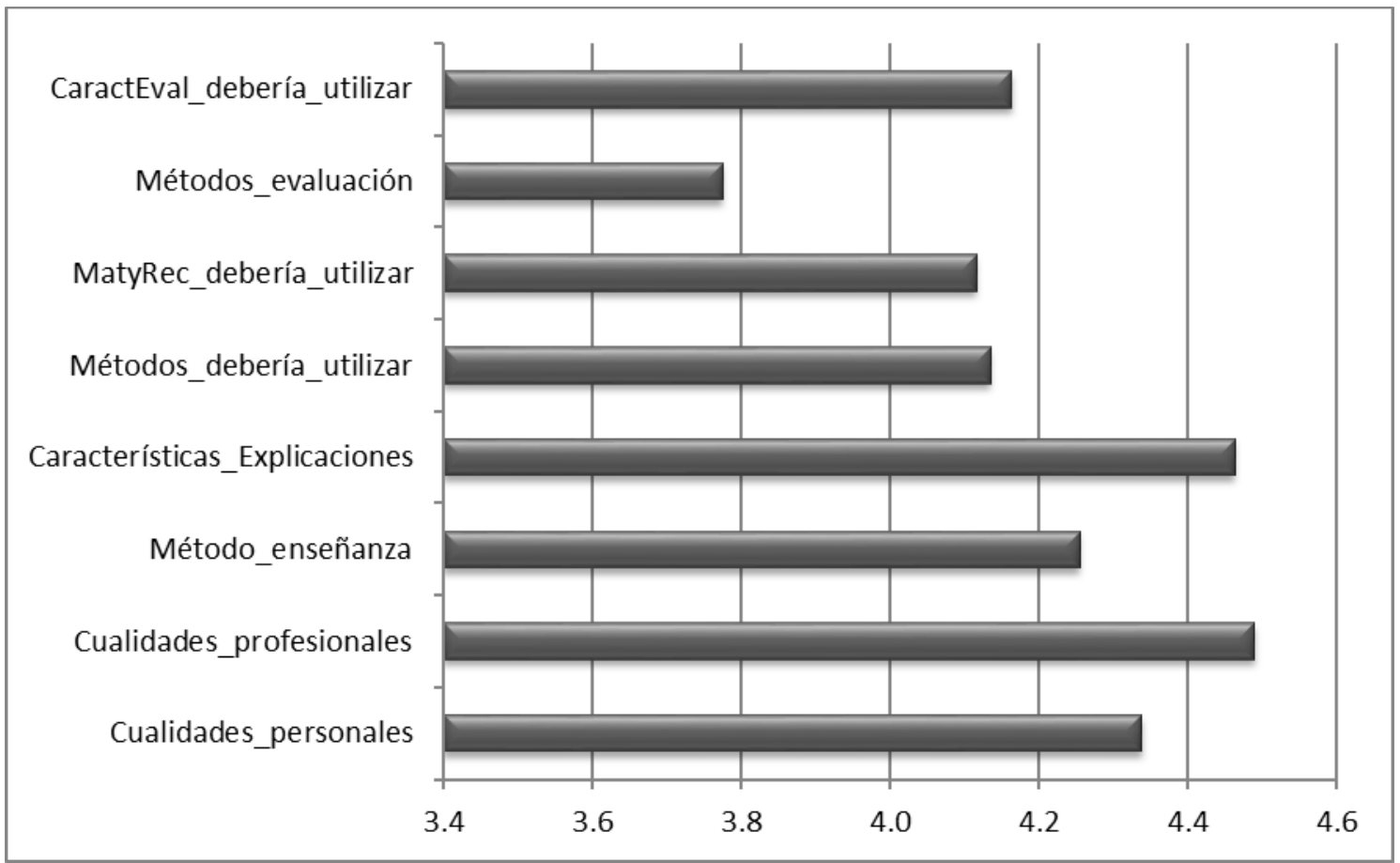

Fuente: Elaboración propia

En la tabla 12 se pueden distinguir si existen diferencias significativas o no, con nivel de significancia de $95 \%$, según el género del encuestado. Los resultados anteriores coinciden con Villalobos-Clavería, Melo-Hermosilla y Perez-Villalobos (2010), quienes muestran en su estudio diferencias significativas en cuanto a que las mujeres presentan expectativas más elevadas que los hombres en torno al proceso educativo. 
Tabla 12. Grado de importancia promedio de las características que definen al buen profesor según el género del estudiante

\begin{tabular}{|l|l|l|l|}
\hline \multicolumn{1}{|c|}{ Dimensión } & \multicolumn{3}{c|}{ Media } \\
\hline \multicolumn{1}{|c|}{ Femenino } & \multicolumn{1}{c|}{ Masculino } & \multicolumn{1}{c|}{ Global } \\
\hline Cualidades profesionales & 4.54 & 4.45 & 4.48 \\
\hline Métodos que debería utilizar & 4.16 & 4.11 & 4.13 \\
\hline $\begin{array}{l}\text { Materiales y recursos didácticos que } \\
\text { debería utilizar }\end{array}$ & 4.12 & 4.11 & 4.11 \\
\hline $\begin{array}{l}\text { Métodos de evaluación } \\
\text { Características de la evaluación que } \\
\text { debería utilizar }\end{array}$ & 4.20 & 4.14 & 4.16 \\
\hline $\begin{array}{l}\text { Cualidades personales del profesor } \\
\text { Características de la metodología de } \\
\text { enseñanza del buen profesor }\end{array}$ & 4.48 & 4.24 & 4.33 \\
\hline $\begin{array}{l}\text { Características de las explicaciones } \\
\text { del buen profesor }\end{array}$ & 4.56 & 4.36 & 4.25 \\
\hline
\end{tabular}

No existen diferencias estadísticamente significativas $(\mathrm{p}<0.05)$

Fuente: Elaboración propia

No se encontraron diferencias significativas, con nivel de confianza de $95 \%$, por especialidad del encuestado en ninguna de las dimensiones, como se muestra en la tabla 13.

Tabla 13. Grado de importancia promedio de las características que definen al buen profesor según la especialidad del estudiante

\begin{tabular}{|l|l|l|l|l|l|l|}
\hline & Pymes & ITI & ITM & IM & ISA & Global \\
\hline Cualidades_personales & 4.4 & 4.6 & 4.3 & 4.2 & 3.8 & 4.3 \\
\hline Cualidades_profesionales & 4.6 & 4.6 & 4.3 & 4.4 & 4.4 & 4.5 \\
\hline Método_enseñanza & 4.4 & 4.4 & 4.0 & 4.1 & 4.2 & 4.3 \\
\hline Características_explicaciones & 4.5 & 4.6 & 4.5 & 4.4 & 4.1 & 4.5 \\
\hline Métodos_debería_utilizar & 4.2 & 4.2 & 3.9 & 4.1 & 4.1 & 4.1 \\
\hline MatyRec_debería_utilizar & 4.1 & 4.3 & 4.0 & 4.0 & 4.1 & 4.1 \\
\hline Métodos_evaluación & 3.7 & 3.8 & 3.8 & 3.8 & 3.8 & 3.8 \\
\hline CaractEval_debería_utilizar & 4.2 & 4.3 & 4.1 & 4.1 & 4.1 & 4.2 \\
\hline
\end{tabular}

No existen diferencias estadísticamente significativas $(\mathrm{p}<0.05)$

Fuente: Elaboración propia 


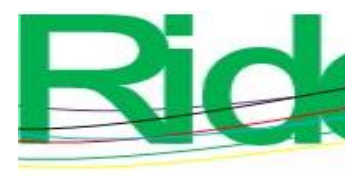

Revista Iberoamericana para la Investigación y el Desarrollo Educativo ISSN 2007 - 7467

La ausencia de diferencias entre programas académicos está relacionada con el hecho de que la percepción depende de la personalidad de los estudiantes, que al estar concentradas sus edades en un rango limitado, sus expectativas de la formación académica y profesional son similares, por lo que la importancia que otorgan a las características del buen profesor no difieren entre sí.

\section{Discusión}

En este estudio se identificaron las cualidades profesionales y las características de las explicaciones como las más importantes para definir a un buen profesor, resultados que coinciden con los presentados por San Martín, Santamaría, Hoyuelos, Ibáñez y Jerónimo (2014), aunque difieren de los presentados por Osuna y Luna (2008), quienes señalan que los alumnos valoran menos la competencia técnica (competencias cognitivas y técnicas) o los hallazgos de Cabalín-Silva et al. (2010), quienes indican como primordiales características la empatía, la responsabilidad y el respeto.

Por otra parte, respecto a las cualidades personales, los resultados coinciden con los obtenidos por Gallardo-López, Sánchez-Peris, Ros-Ros y Ferreras-Remesal (2010), para quien el respeto y la capacidad de escucha son características fundamentales de la personalidad del buen profesor, y con Luna-Serrano, Valle-Espinosa y Osuna-Lever (2010), quienes apuntan que las menos importantes son las competencias sociales. Asimismo, se difiere con Cabalín-Silva et al. (2010) en que la empatía es un atributo principal, mientras que en cualidades profesionales los resultados se equiparan con los obtenidos por Santos-Guerra (1991) al igual que HamerFlores (2015), Tarabay-Yunes (2009) y Merellano-Navarro et al. (2016), quienes coinciden en destacar que el profesor debe ser un conocedor de la disciplina que desarrolla, un especialista en su rama.

Sobre la metodología de enseñanza, los estudiantes calificaron con mayor importancia el establecer relaciones entre los conceptos y temas de la asignatura. Esto concuerda con Gallardo-López et al. (2010) y Cardona-Rodríguez et al. (2009), quienes explican que se debe fomentar el aprendizaje significativo. En consecuencia, el docente pasa de ser un trasmisor de conocimientos a un facilitador que busca estrategias de enseñanza para hacer frente a la diversidad de requerimientos sociales y productivos de los estudiantes. El propósito es reducir la clase magistral (puntuación más baja según la percepción de los estudiantes) y promover la utilización de metodologías adecuadas para consolidar el conocimiento (Sayós et al., 2014). 


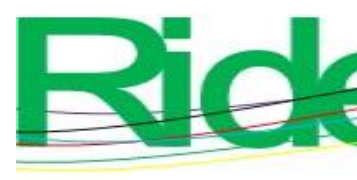

Revista Iberoamericana para la Investigación y el Desarrollo Educativo ISSN 2007 - 7467

Otro aspecto calificado de forma negativa fue el promover el trabajo individual del alumno, pues en el aula se prefiere una dinámica participativa (Casero-Martínez, 2010). Sin embargo, se difiere con Luna-Serrano et al. (2010), quienes señalan que lo menos importante es el trabajo en equipo.

Al igual que Tarabay-Yunes (2009), Casero-Martínez (2010), Abadía-Valle et al. (2015) y Merellano-Navarro et al. (2016), los resultados indican que para ser un buen profesor se deben explicar de forma clara y ordenada los contenidos de la asignatura. De hecho, el estudiante debe concebir el aprendizaje como algo emocional, y no solo cognitivo, utilizando explicaciones motivadoras (Gallardo-López et al., 2010). Además, el buen profesor debe asesorar, orientar (Ruiz-Bueno et al. 2008) y definir las estrategias de intervención y apoyo oportuno según cada contexto (Santos-Guerra, 1991).

Por otra parte, es imperante incluir en el ejercicio de la enseñanza una didáctica profesional pensada para el alumno y según los requerimientos de la asignatura, con suficientes herramientas didácticas y tecnologías (Núñez-Rodríguez, Fajardo-Ramos y Químbayo-Díaz, 2010; Rocha-Chávez, 2012). En el análisis realizado en esta investigación, los materiales de estudio claros y sencillos resultan de mayor importancia para los estudiantes.

En cuanto a los métodos de evaluación, para los estudiantes encuestados es primordial la valoración que da el profesor a la realización de prácticas y al esfuerzo del alumno, lo que coindice con lo señalado por Hamer-Flores (2015) y Gallardo-López et al. (2010). De hecho, resultó de menor importancia dentro de los métodos de evaluación la aplicación de un solo examen final, lo que también coincide con lo expuesto por San-Martín et al. (2014). La valoración de la asistencia tanto a clase como a tutorías y asesorías para la resolución de dudas son aspectos poco valorados. En otras palabras, si el alumno aprende en el salón de clase, no requiere asesoría y la asistencia no es determinante en el nivel de aprendizaje del alumno. Esto significa que se debe mostrar justicia y coherencia a la hora de diseñar los criterios de evaluación y revisión (Casero-Martínez, 2010).

En la evaluación, el docente juega el rol de líder directivo de cada asignatura, pues es quien asume la conducción y claridad de los criterios valorados para el logro de las metas y los objetivos, lo cual evitaría conflictos ocasionados por la omisión de información a los estudiantes (Cardona-Rodríguez et al., 2009).

Finalmente, una de las principales limitaciones del presente estudio fue que se realizó solo con estudiantes del último ciclo escolar; por tanto, se puede desarrollar una investigación comparativa entre los demás ciclos para detectar diferencias y similitudes en cuanto a los criterios valorados por los estudiantes en cuanto al perfil de un buen profesor. 


\section{Conclusiones}

El objetivo del presente estudio se logró al identificar las características y cualidades que caracterizan al buen profesor según la percepción del alumno del último ciclo de formación de la universidad politécnica objeto de análisis. Asimismo, las áreas de oportunidad detectadas fueron múltiples, por lo que cada docente tendrá la posibilidad de contrastar los resultados aquí enseñados con su propia práctica pedagógica. Si él cuenta con algunas de las características que hacen del docente un buen profesor, deberá seguir trabajando y fomentando esas fortalezas a través de cursos y talleres de capacitación, lo que redundará en la optimización de su proceso de enseñanza-aprendizaje. De hecho, la confirmación de la hipótesis planteada señala la importancia de promover la competencia profesional del profesor, factor clave para desempeñar con éxito la tarea educativa.

\section{Futuras Líneas de Investigación}

Finalmente, se sugieren las siguientes líneas de investigación: un análisis (esta vez desde la perspectiva del docente) de las cualidades que definen a un buen profesor. Asimismo, un estudio comparativo del impacto, desde la perspectiva del estudiante, de las funciones básicas de la universidad y la participación del profesor en ellas: docencia, proyección social e investigación; el propósito de este análisis sería identificar qué tan importante consideran los alumnos que el profesor universitario desarrolle estas funciones, especialmente la investigación y proyección social como un medio de actualización y mejora de su práctica docente. 


\section{Referencias}

Abadía-Valle, A. R., Bueno-García, C., Ubieto-Artur, M. I., Márquez-Cebrián, M. D., SabatéDíaz, S., Jorba-Noruega, H. y Pagés-Costa, T. (2015). Competencias del buen docente universitario: opinión de los estudiantes. Revista de Docencia Universitaria, 13(2), 363390. Doi: http://dx.doi.org/10.4995/redu.2015.5453

Cabalín-Silva, D., Navarro-Hernández, N. y Zamora-Silva, J. (2010). Concepción de estudiantes y docentes del buen profesor universitario: Facultad de Medicina de la Universidad de la Frontera. International Journal of Morphology, 28(1), 283-290. Doi: http://dx.doi.org/10.4067/S0717-95022010000100042

Cardona-Rodríguez, A., Barrenetxea-Ayesta, M., Mijangos-del-Campo, J. J. y OlaskoagaLarrauri, J. (2009). Concepto y determinantes de la calidad de la educación superior. Un sondeo de opinión entre profesores de universidades españolas. Archivos Analíticos de Políticas Educativas, 17(10). Recuperado de http://epaa.asu.edu/epaa/

Casero-Martínez, A. (2010). ¿Cómo es el buen profesor universitario según el alumnado?

Revista Española de Pedagogía, 68(246), 223-242. Recuperado de http://revistadepedagogia.org/index.php/es/ano-2010/61-no-246-mayo-agosto2010/414-icomo-es-el-buen-profesor-universitario-segun-el-alumnado

Diario Oficial de la Federación [DOF] (2021). Ley Federal de Educación. Recuperado de https://www.dof.gob.mx/nota_detalle.php?codigo=5573858\&fecha=30/09/2019

Gallardo-López, B., Sánchez-Peris, F., Ros-Ros, C. y Ferreras-Remesal, A. (2010). Estilos docentes de los profesores universitarios: la percepción de los buenos profesores Facultad de Filosofía y Ciencia de la Universidad de Valencia, España. Revista Iberoamericana de Educación, 51(4). Recuperado de http://rieoei.org/3236.htm

Gutiérrez, D. (2017). Buenas prácticas desde la percepción de los estudiantes de la licenciatura en educación para el medio indígena. Educación y Humanismo, 20(34), 258-270. Doi: https://doi.org/10.17081/eduhum.20.34.2869

Hamer-Flores, A. (2015). La percepción del buen profesor en alumnos de nuevo ingreso a la enseñanza universitaria: el caso de ETEA (Córdoba). Revista Complutense de Educación, 26(2), 227-240.

Recuperado de http://revistas.ucm.es/index.php/RCED/article/view/41534

Hickman, H., Alarcón, M., Cepeda, M., Cabrera, R. y Torres, X. (2016). Significado de buen profesor y de evaluación docente por estudiantes y maestros universitarios. La técnica de redes semánticas. Sinéctica, (47). Recuperado de https://sinectica.iteso.mx/index.php/SINECTICA/article/view/636 
Luna-Serrano, E., Valle-Espinosa, M. C. y Osuna-Lever, C. (2010). Los rasgos de un "buen profesional", según la opinión de estudiantes universitarios en México. Revista Electrónica de Investigación Educativa, 12. Recuperado de http://redie.uabc.mx/redie/article/view/254

Merellano-Navarro, E., Almonacid-Fierro, A., Moreno-Doña, A. y Casto-Jaque, C. (2016). Buenos docentes universitarios. ¿Qué dicen los estudiantes? Educação e Pesquisa, 42(4), 937-952. Doi: http://dx.doi.org/10.1590/S1517-9702201612152689

Miranda, C. (2007). Educación superior, mecanismos de aseguramiento de la calidad y formación docente: un debate pendiente en Chile. Estudios Pedagógicos 33(1), 95-108. Recuperado de http://mingaonline.uach.cl/scielo.php?script=sci_abstract\&pid=S071807052007000100006\&lng=es\&nrm=iso\&tlng=es

Nowakowski, P. (2007). Trabajo del profesorado universitario según lo evalúan los estudiantes. Educação, 30(63), $\quad$ 547-556. Recuperado de http://revistaseletronicas.pucrs.br/ojs/index.php/faced/article/view/2749

Núñez-Rodríguez, M. L., Fajardo-Ramos, E. y Químbayo-Díaz, J. H. (2010). El docente como motivador: percepciones de los estudiantes de la Facultad de Ciencias de la Salud de la Universidad de Tolima (Colombia). Salud Uninorte, 26(2), 260-268. Recuperado de http://rcientificas.uninorte.edu.co/index.php/salud/article/viewArticle/990/5810

Organización de las Naciones Unidas [ONU] (2015). Asamblea General-Transformar nuestro mundo: la Agenda 2030 para el desarrollo sostenible. Nueva York: ONU. Recuperado de http://www.un.org/es/comun/docs/?symbol=A

Organización de las Naciones Unidas para la Educación, la Ciencia y la Cultura [Unesco]. (2015). Guía para el desarrollo de políticas docentes. París: Unesco.

Organización de las Naciones Unidas para la Educación, la Ciencia y la Cultura [Unesco] (2020). La educación transforma vidas. Recuperado de https://es.unesco.org/themes/education

Osuna, C. y Luna, E. (2008). Características de ser un buen profesional de Ingeniería en la Universidad Autónoma de Baja California, México. Formación Universitaria, 1(1), 2936. Doi: 10.4067/S0718-50062008000100005

Rocha-Chávez, R. (2012). La docencia universitaria desde la perspectiva de los alumnos frente a la de los profesores. Innovación Educativa, 12(58), 91-118. Recuperado de http://www.innovacion.ipn.mx/Revistas/Paginas/Revista-Innovacion-Educativa-58.aspx Ruiz-Bueno, C., Mas-Torelló, O., Tejeda-Fernández, J. y Navío-Gámez, A. (2008). Funciones y escenarios de actuación del profesor universitario. Apuntes para la definición del perfil basado en competencias. Revista de la Educación Superior, 37(2). 115-132. Recuperado 
de http://publicaciones.anuies.mx/revista/146/4/2/es/funciones-y-escenarios-deactuacion-del-profesor-universitario

San-Martín, S., Santamaría, M., Hoyuelos, F. J., Ibáñez, J. y Jerónimo, E. (2014). Variables definitorias del perfil del profesor/a universitario/a ideal desde la perspectiva de los estudiantes preuniversitarios/as. Educación XX1, 17(2), 193-215. Doi: 10.5944/educxx1.17.1.11486

Santos-Guerra, M (1991). Criterios de referencia sobre calidad de proceso de enseñanza/aprendizaje en la universidad. Revista de Enseñanza Universitaria, 1(1), 2547. Recuperado de http://institucional.us.es/revistas/universitaria/1/art_3.pdf

Sayós, R., Pagés, T., Amador, J. A. y Jorba, H (2014). Ser buen docente: ¿qué opinan los estudiantes de la Universidad de Barcelona? Revista Iberoamericana de Psicología y Salud, 5(2), 135-149. Recuperado de https://dialnet.unirioja.es/servlet/articulo?codigo=4762654

Secretaría de Educación Pública [SEP] (2011). Educación por niveles. http://www.sep.gob.mx/es/sep1/educacion_por_niveles

Tarabay-Yunes, F. (2009). Cualidades docentes universitarias: de la pedagogía a la relación afectivo-comunicativa. Testimonios de estudiantes universitarios. Revista de Teoría y Didáctica de las Ciencias Sociales, 15, 355-377. Recuperado de http://www.saber.ula.ve/handle/123456789/31190

Torres-Rivera, A. D., Badillo-Gaona, M., Valentin-Kajatt, N. O. y Ramírez-Martínez, E. T. (2014). Las competencias docentes: el desafío de la educación superior. Innovación $\begin{array}{llll}\text { Educativa, } & \text { 14(66), } & \text { 129-146. }\end{array}$ http://www.innovacion.ipn.mx/Revistas/Paginas/revista-innovacion-educativa-no66.aspx

Universidad Politécnica de Victoria [UPV] (2016). Plan de Desarrollo Institucional 2013-2020. Recuperado de http://www.upvictoria.edu.mx/pdi/

Vaillant, D. y Siqueira, V. (2017). Estudiantes de ingeniería y sus percepciones sobre la enseñanza de calidad. Actualidades Investigativas en Educación, 17(3), 1-19. Recuperado de https://www.redalyc.org/articulo.oa?id=447/44758585020

Valencia, L. (2019). Los buenos profesores: un ensayo crítico-literario sobre un arte siempre en peligro. Acta Scientiarum. Education, 41. Recuperado de https://www.redalyc.org/articulo.oa?id=3033/303360435008

Villalobos-Clavería, A., Melo-Hermosilla, Y. y Perez-Villalobos, C. (2010). Percepción y expectativas de los alumnos universitarios frente al profesor no pedagogo. Estudios $\begin{array}{llll}\text { Pedagógicos, } & 36(2), & \text { 241-249. } & \text { Recuperado }\end{array}$ 
http://mingaonline.uach.cl/scielo.php?script=sci_abstract\&pid=S0718-

07052010000200014\&lng=es\&nrm=iso\&tlng=es

Yurén, T., García-García, F., Escalante Ferrer, A., González-Barrera, Z. y Velázquez Albavera, D. (2020). La representación del buen docente universitario entre dos enfoques: transmisivo y constructivista. Revista Mexicana de Investigación Educativa, 25(85), 239265. Recuperado de https://www.redalyc.org/articulo.oa?id=140/14064761002 


\begin{tabular}{|l|l|}
\hline Rol de Contribución & Autor (es) \\
\hline Conceptualización & Estela Torres Ramírez, Daniela Cruz Delgado, igual \\
\hline Metodología & Daniela Cruz Delgado \\
\hline Software & No aplica \\
\hline Validación & Juan López Hernández \\
\hline Análisis Formal & Daniela Cruz Delgado \\
\hline Investigación & $\begin{array}{l}\text { Juan López Hernández (principal), Juan Enrique Lira Uribe } \\
\text { (apoya) }\end{array}$ \\
\hline Recursos & Estela Torres Ramírez \\
\hline Curación de datos & Daniela Cruz Delgado \\
\hline $\begin{array}{l}\text { Escritura - Preparación del } \\
\text { borrador original }\end{array}$ & $\begin{array}{l}\text { Daniela Cruz Delgado (Resultados y discusión), Estela Torres } \\
\text { Ramírez (Marco teórico), Juan López Hernández (Discusión de } \\
\text { resultados y conclusiones), Juan Enrique Lira Uribe (Marco } \\
\text { teórico) }\end{array}$ \\
\hline $\begin{array}{l}\text { Escritura - Revisión y } \\
\text { edición }\end{array}$ & \begin{tabular}{l} 
Daniela Cruz Delgado, Juan López Hernández, igual \\
\hline Visualización
\end{tabular} \\
\hline Supervisión & Daniela Cruz Delgado \\
\hline Administración de Proyectos & Daniela Cruz Delgado \\
\hline Adquisición de fondos & Estela Torres Ramírez \\
\hline
\end{tabular}

